\title{
First-principles calculations of phase transition, elasticity, and thermodynamic properties for TiZr alloy
}

\author{
Bao-Tian Wang, ${ }^{1,2}$ Wei-Dong $\mathrm{Li}^{1}{ }^{1}$ and Ping Zhang ${ }^{2,3}$, 因 \\ ${ }^{1}$ Institute of Theoretical Physics and Department of Physics, \\ Shanxi University, Taiyuan 030006, People's Republic of China \\ ${ }^{2}$ LCP, Institute of Applied Physics and Computational Mathematics, Beijing 100088, People's Republic of China \\ ${ }^{3}$ Center for Applied Physics and Technology, Peking University, Beijing 100871, People's Republic of China
}

\begin{abstract}
Structural transformation, pressure dependent elasticity behaviors, phonon, and thermodynamic properties of the equiatomic TiZr alloy are investigated by using first-principles density-functional theory. Our calculated lattice parameters and equation of state for $\alpha$ and $\omega$ phases as well as the phase transition sequence of $\alpha \rightarrow \omega \rightarrow \beta$ are consistent well with experiments. Elastic constants of $\alpha$ and $\omega$ phases indicate that they are mechanically stable. For cubic $\beta$ phase, however, it is mechanically unstable at zero pressure and the critical pressure for its mechanical stability is predicted to equal to $2.19 \mathrm{GPa}$. We find that the moduli, elastic sound velocities, and Debye temperature all increase with pressure for three phases of TiZr alloy. The relatively large $B / G$ values illustrate that the $\mathrm{TiZr}$ alloy is rather ductile and its ductility is more predominant than that of element $\mathrm{Zr}$, especially in $\beta$ phase. Elastic wave velocities and Debye temperature have abrupt increase behaviors upon the $\alpha \rightarrow \omega$ transition at around $10 \mathrm{GPa}$ and exhibit abrupt decrease feature upon the $\omega \rightarrow \beta$ transition at higher pressure. Through Mulliken population analysis, we illustrate that the increase of the $d$-band occupancy will stabilize the cubic $\beta$ phase. Phonon dispersions for three phases of TiZr alloy are firstly presented and the $\beta$ phase phonons clearly indicate its dynamically unstable nature under ambient condition. Thermodynamics of Gibbs free energy, entropy, and heat capacity are obtained by quasiharmonic approximation and Debye model.
\end{abstract}

PACS numbers: $62.20 . \mathrm{de}, 64.70 . \mathrm{kd}, 61.50 . \mathrm{Ks}, 74.70 . \mathrm{Ad}$

\section{INTRODUCTION}

Group IV transition metals such as titanium, zirconium, and hafnium as well as their alloys have been investigated extensively by experiments and theoretical calculations since their particular applications in the aerospace, nuclear, and chemical industries [1-4]. Their properties of low thermal neutron absorption cross section, adequate strength and ductility, good corrosion resistance, long-term dimensional stability in an irradiation environment, excellent compatibility with the fuel and coolant, good thermal conductivity and adequate resistance to fracture project them to the front of material applications for both fuel element cans and in-core structural components in water-cooled nuclear reactors 5]. Specifically, TiZr alloy is traditionally used for highpressure neutron diffraction and has similar machining properties to the ZrNb alloy used in nuclear-reactor tubing. Therefore, investigations of their structural and elastic properties, which relate to various fundamental solidstate properties such as interatomic potentials, equation of state, phonon spectra, and thermodynamic properties, have prominent meaning in both scientific and technological fields. The scientific interest in Group IV transition metals and their alloys origins from the fact that they have a narrow $d$-band in the midst of a broad $s p$-band. The $d$-band occupancy is crucial for the structural stabil-

\footnotetext{
*Corresponding author; zhang-ping@iapcm.ac.cn
}

ity of these materials and a pressure-induced $s$ - $d$ electron transfer is the driving force behind their structural and electronic transitions [6, 7].

At ambient condition, both $\mathrm{Ti}$ and $\mathrm{Zr}$ crystallize in hexagonal closed-packed (hcp) structure ( $\alpha$ phase). At high temperature of 1155 and $1135 \mathrm{~K}$, they transform into the body-centered cubic (bcc) $\beta$ phase, respectively. At room temperature and under compression, $\alpha$ $\mathrm{Zr}$ transforms to another hexagonal structure $\omega$ phase at about $2-7 \mathrm{GPa}[8-11]$. Under further high pressure of $30-35 \mathrm{GPa}$, the $\omega \rightarrow \beta$ phase transition has been observed [10, 12 15]. The whole $\alpha \rightarrow \omega \rightarrow \beta$ transition series for $\mathrm{Zr}$ have been reproduced in theoretical investigations [16 19]. However, the experimental established room temperature transition sequence of $\mathrm{Ti}$ is $\alpha \rightarrow \omega \rightarrow \gamma \rightarrow \delta$ [4, 10, 20, 21]. The $\beta$ phase of Ti metal has not been observed up to $216 \mathrm{GPa} 20$. Recent first-principles study performed by Mei et al. 22] found that the $\delta$-Ti is not stable under hydrostatic compression and the $0 \mathrm{~K}$ phase transition sequence of $\mathrm{Ti}$ is $\alpha \rightarrow \omega \rightarrow \gamma \rightarrow \beta$. The absence of the high-pressure $\beta$ phase for Ti in experiments was attributed to the possible nonhydrostatic stress which distorts the $\beta$ phase [23].

For Ti-Zr alloys, transition sequence of $\alpha \rightarrow \omega \rightarrow \beta$ upon compression has been reported by Bashkin et al. [24 26. . Using the differential thermal analysis (DTA) and calorimetric technique, they extensively studied the effects of temperature and pressure on the $\alpha \rightleftarrows \omega$ and $\omega \rightleftarrows \beta$ transformations in the equiatomic TiZr alloy at temperatures up to $1023 \mathrm{~K}$, and pressures up to $7 \mathrm{GPa}$. 24] At atmospheric pressure, the $\beta \rightleftarrows \alpha$ transition tempera- 
ture was measured to be $852 \mathrm{~K}$ upon cooling. Under pressure, the transition temperature decreases down to the triple $\alpha-\beta-\omega$ equilibrium point $\left(P_{\mathrm{tr}}=4.9 \pm 0.3 \mathrm{GPa}\right.$, $\left.T_{\mathrm{tr}}=733 \pm 30 \mathrm{~K}\right)$. At pressures above the triple point, the slope of the $\omega-\beta$ equilibrium boundary is positive at pressures up to $7 \mathrm{GPa}$. At room temperature, the $\alpha \rightleftarrows \omega$ transition occurs at around $5 \mathrm{GPa}$. Cooling of the $\beta$ phase in the pressure range $2.8-4.8 \mathrm{GPa}$ can form a twophase mixture of a stable $\alpha$ and a metastable $\omega$ phase. Another group [27] reported that the equilibrium $\alpha-\omega$ boundary is situated on the $P-T$ diagram at $6.6 \mathrm{GPa}$. Afterward, Bashkin et al. [25, 26] employed the energydispersive X-ray diffraction (EDXD) with synchrotron radiation to investigate the structural transitions of the TiZr alloy. They found an increase in the $\omega \rightarrow \beta$ transition pressure from about 30 to $43-57 \mathrm{GPa}$ when the titanium content in the alloys increases from 0 to 50 at. $\%$. The pressure dependant behaviors of superconductivity was also presented. For equiatomic TiZr alloy, they found that the $\alpha$ phase remains the sole stable phase under quasi-hydrostatic pressure up to $12.2 \mathrm{GPa}$ [26]. Only from 15.5 GPa on, the $\omega$ phase becomes dominant. Recent in situ high-temperature high-pressure angle-dispersive synchrotron radiation diffraction experiment revealed that under pressure the equiatomic $\mathrm{TiZr}$ alloy occurs an $\omega \rightarrow \omega^{\prime}$ isostructural transition at highpressure domain [28].

However, to date only one theoretical study has focused on TiZr alloy [29], where they calculated the electron structure and total energy by the scalar relativistic full-potential linearized augmented-planewave (FPLAPW) method with a generalized gradient approximation (GGA) potential. The $P-T$ phase diagram and tendency toward decomposition in equiatomic TiZr alloy were calculated within the electron density functional theory (DFT) and the Debye-Grüneisen model. Their calculations showed that the $\omega$ phase is stable at atmospheric pressure and moderate (up to $610 \mathrm{~K}$ ) temperatures, and hence no $\alpha \rightarrow \omega$ transition occurs at room temperature. They attributed the discrepancy between experiment and theory to the experimental samples, within which defects may exist. In present work, we have carefully calculated the structural, phase transition, elastical, phonon vibrational, and thermodynamic properties for equiatomic TiZr alloy by employing the first-principles total energy calculations. Elastic constants, elastic properties, and phonon dispersions of three experimentally observed phases are firstly presented. Our main motivation is to give out detail pressure behaviors of the elasticity as well as the thermodynamic properties to support the practical application of TiZr alloy in nuclear technology.

\section{COMPUTATIONAL METHODS}

First-principles DFT calculations on the basis of the frozen-core projected augmented wave (PAW) method of Blöchl [30] are performed within the Vienna $a b$ initio simulation package (VASP) [31], where the Perdew, Burke, and Ernzerhof (PBE) 32] form of the GGA is employed to describe electron exchange and correlation. To obtain accurate total energy and stress tensor, a cutoff energy of $500 \mathrm{eV}$ is used for the plane-wave set. The $\Gamma$-centered $k$ point-meshes in the full wedge of the Brillouin zone (BZ) are sampled by $18 \times 18 \times 16,16 \times 16 \times 9$, and $18 \times 18 \times 18$ grids according to the Monkhorst-Pack (MP) 33] for $\alpha$ (two atoms cell), $\omega$ (six atoms $1 \times 1 \times 2$ supercell), and $\beta$ (two atoms cell) phases TiZr alloy, respectively. Full geometry optimization at each volume is considered to be completed when all atoms are fully relaxed until the Hellmann-Feynman forces becoming less than $0.001 \mathrm{eV} / \AA$. The Ti $3 s^{2} 3 p^{6} 3 d^{3} 4 s^{1}$ and the Zr $4 s^{2} 4 p^{6} 4 d^{3} 5 s^{1}$ orbitals are explicitly included as valence electrons.

\section{RESULTS}

\section{A. Structure}

In calculations of $\alpha$ phase TiZr alloy, we use one lamella structure, which has the same unit cell as the conventional hcp unit cell but the $\mathrm{Ti}$ and $\mathrm{Zr}$ atoms are located on the layer along the $c$ axis alternately. For $\beta$ phase, $\mathrm{Ti}$ atoms are fixed at corner and $\mathrm{Zr}$ atoms are located at center of the bcc unit cells. For $\omega$ TiZr, two types (Atype and B-type) of $1 \times 1 \times 2$ supercells are constructed (Fig. 1). The theoretical equilibrium structural parameters, bulk modulus $B$, and pressure derivative of the bulk modulus $B^{\prime}$ obtained by fitting the energy-volume data in the third-order Birch-Murnaghan equation of state (EOS) [34] are tabulated in Table 1. Experimental values from Ref. [26] and other theoretical results from Refs. [1] and [29] are also presented for comparison. Note that we only list in Table 1 the results of B-type structure for $\omega$ phase. Our calculated equilibrium structural parameters for A-type $\omega$ phase are $a_{0}=4.829 \AA$ and $c_{0}=3.013 \AA$. Its bulk modulus $B$ and pressure derivative of the bulk modulus $B^{\prime}$ are equal to $100.6 \mathrm{GPa}$ and 3.63, respectively. All these values are almost equal to that of B-type structure. However, the total energy of B-type structure is smaller by $0.0769 \mathrm{eV}$ per formula unit than that of A-type structure. Using A-type structure for $\omega$ phase will lead to incorrect results in calculations of phase transition and mechanical properties. Thus, the results of A-type $\omega$ phase are not presented in the following and unless otherwise stated results of $\omega$ phase refer to that of B-type structure.

From Table 1, one can find excellent coincidence between our calculated values and the corresponding experimental results for $\alpha$ and $\omega$ structural parameters. But our calculated bulk moduli $B$ for these two phases are largely smaller than that from experiment. Although we can not simply attribute these results to the underbinding effect of the GGA approach, we find that our 
TABLE I: Calculated lattice parameters ( $a$ or $c$ ), bulk modulus $(B)$, pressure derivative of the bulk modulus $\left(B^{\prime}\right)$, and elastic constants of $\alpha, \omega$, and $\beta$ TiZr at different pressures. For comparison, experimental values and other theoretical results are also listed.

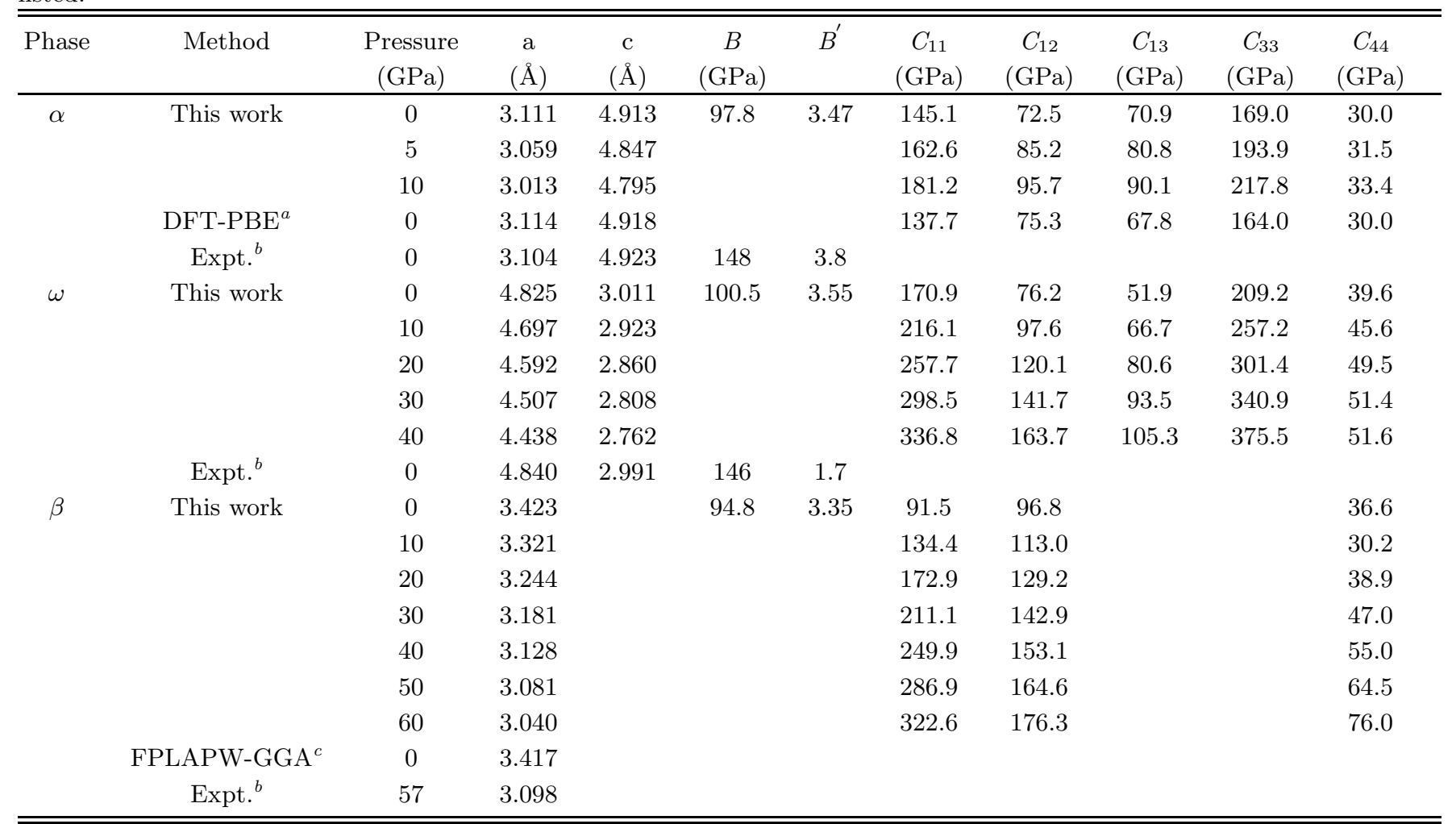

${ }^{a}$ Reference [1], ${ }^{b}$ Reference [26], ${ }^{c}$ Reference [29].

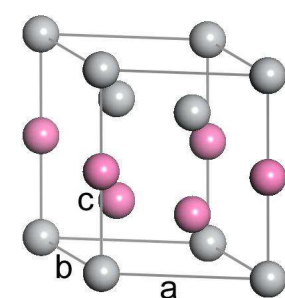

(a)

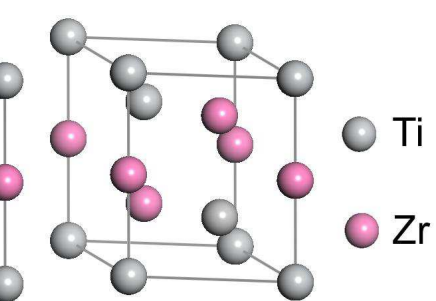

(b)
FIG. 1: (Color online) Schematic pictures of $1 \times 1 \times 2$ supercells for $\omega$ phase in (a) A-type and (b) B-type structures.

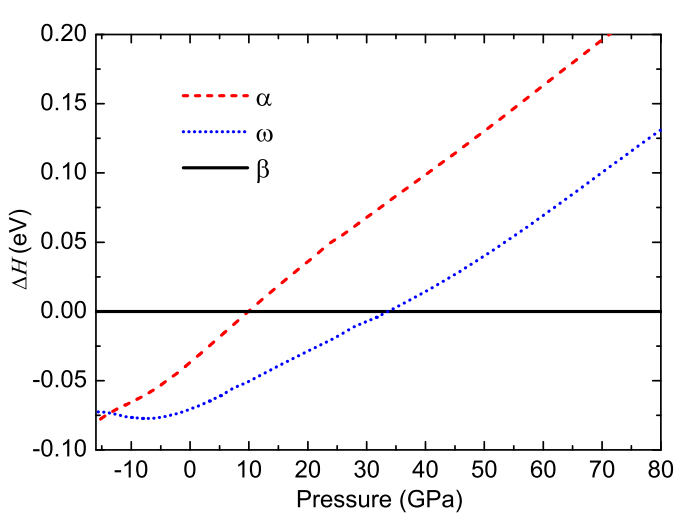

FIG. 2: (Color online) Calculated enthalpy differences of $\alpha$ and $\omega$ phases with respect to $\beta$ phase as a function of pressure.

\section{B. Phase transition at $0 \mathrm{~K}$}

the range of experimental values between Ti $102 \mathrm{GPa}[4]$ and $\mathrm{Zr} 92 \mathrm{GPa}[11]$. As for $\beta$ phase, our calculated equilibrium crystal constant is in good agreement with recent theoretical calculation [29], where a value of $3.417 \AA$ was given out. The discrepancy between our results and the experimental value $(3.417 \AA$ at $57 \mathrm{GPa})$ [26] mainly due to the temperature effect. 
TABLE II: Calculated transition pressure for TiZr. For comparison, other theoretical works and available experimental data for $\mathrm{TiZr}, \mathrm{Ti}$, and $\mathrm{Zr}$ are listed.

\begin{tabular}{llcr}
\hline \hline & & \multicolumn{2}{c}{$\begin{array}{c}\text { Transition pressure } \\
\text { (GPa) }\end{array}$} \\
\cline { 3 - 4 } Metal & Transition & Expt. & Theory \\
\hline TiZr & $\alpha \rightarrow \omega$ & $5-11^{a}$ & $-13.8^{b}$ \\
& $\omega \rightarrow \beta$ & $43^{a}$ & $26^{c}, 33.9^{b}$ \\
$\operatorname{Ti}$ & $\alpha \rightarrow \omega$ & $2-11.9^{d}$ & $52^{e},-3.7^{f}$ \\
$\mathrm{Zr}$ & $\alpha \rightarrow \omega$ & $2-7^{g}$ & $4.8^{h}, 3.3^{i},-3.7^{j}$ \\
& $\omega \rightarrow \beta$ & $30-35^{k}$ & $32.4^{j}$ \\
\hline \hline
\end{tabular}

${ }^{a}$ Reference 24, 26, 27], ${ }^{b}$ Present work, ${ }^{c}$ References [29], ${ }^{d}$ References [4, 10, 20, 21], ${ }^{e}$ Reference [39], ${ }^{f}$ Reference [22], ${ }^{g}$ References [9 11], ${ }^{h}$ Reference 35], ${ }^{i}$ Reference [36], ${ }^{j}$ Reference [16], ${ }^{k}$ References [10, 12 15].

at ambient pressure the $\omega$ phase is more stable than the $\alpha$ phase. This fact is in disagreement with experiment [24, 26, 27], but coincides with recent theoretical calculations [29]. Trubitsin et al. [29] attributed the discrepancy between experiment and theory to the experimental impure samples. They presented detailed explanation for this discrepancy. In our opinion, the imperfect crystals used in experiments may responsible mainly for this discrepancy. After all, the metastable $\omega$ phase was obtained at atmospheric pressure through cooling of the $\beta$ phase under a pressure of $6 \mathrm{GPa}$ with subsequent unloading at room temperature. One other reason may from the temperature contribution. In Table 2, we list the transition pressures for TiZr alloy as well as pure Ti and $\mathrm{Zr}$ metals from experiments and theoretical calculations. For the stable phase of metals $\mathrm{Ti}$ and $\mathrm{Zr}$ at ambient pressure, there exist debates in theoretical studies [16, 17, 22, 3541] although experiments [4, 9-11, 20] have reported the most stable phase to be $\alpha$ phase. For metal Zr, two DFT works 35, 36 using full-potential linear muffin-tin orbital (FPLMTO)-GGA have illustrated the $\alpha \rightarrow \omega$ transition for $\mathrm{Zr}$ at 4.8 and $3.3 \mathrm{GPa}$, respectively. However, two other DFT works [37, 38] using FPLMTO-GGA and FPLAPW-LDA methods indicated that the most stable phase at $0 \mathrm{~K}$ under ambient pressure is $\omega$, not $\alpha$ phase. Two recent DFT-PBE works [16, 17] also support this conclusion. For metal Ti, the debates also exist in DFT calculations [22, 39] as indicated in Table II. By adding the phonon contribution to the Gibbs energy, recent DFT-PBE [17, 42] studies explicitly showed that the transition of $\alpha \rightarrow \omega$ for $\mathrm{Ti}$ (Zr) occurs at 1.8 (1.7) GPa at room temperature. Thus, the disagreement between some theoretical works and experiments for elemental $\mathrm{Ti}$ and $\mathrm{Zr}$ mainly originates from temperature effect. The entropy from the thermal population of phonon states will stabilize the $\alpha$ phase at room temperature 22]. In our present work, we should also consider the temperature effect on the $\alpha \rightarrow \omega$ transition pressure for TiZr alloy since the same scheme used with respect to our previ-

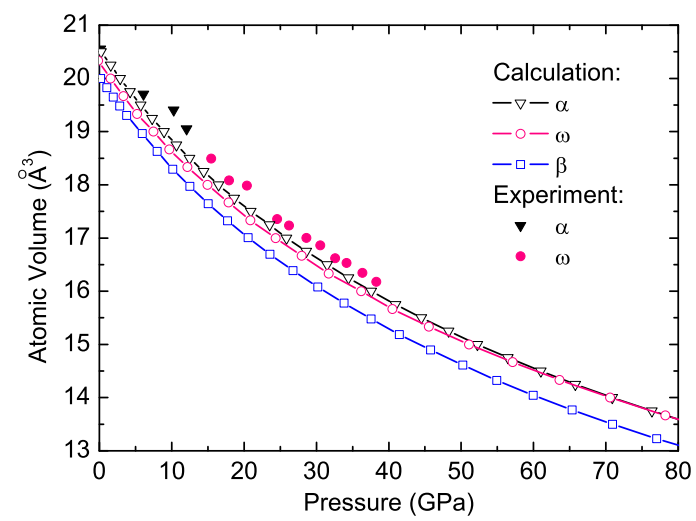

FIG. 3: (Color online) Calculated compression curves of TiZr alloy compared with experimental measurements from Ref. $[25]$.

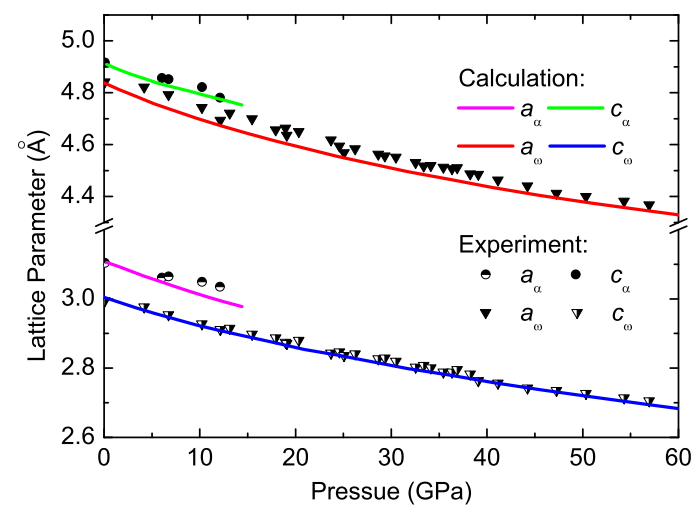

FIG. 4: (Color online) Calculated lattice parameters in comparison with the experimental data of Bashkin et al. [26] for $\alpha$ and $\omega$ phases of TiZr alloy. Our calculated results of $\omega^{\prime}$ phase are also presented.

ous study of $\mathrm{Zr}$ [16]. In addition, Trubitsin et al. 29] observed that $\alpha$ TiZr alloy is stable in the temperature range $600 \mathrm{~K}<\mathrm{T}<900 \mathrm{~K}$ after including lattice energy and entropy in the Debye model. Therefore, the disagreement of $\alpha \rightarrow \omega$ transition pressure between our calculation and experiment for TiZr alloy can also be attributed mainly to the experimental impure samples and partially to the temperature effect. As for the $\omega \rightarrow \beta$ transition, our calculated transition pressure $(33.9 \mathrm{GPa})$ is in good agreement with experiment [26].

To further analyze the pressure behavior of TiZr alloy, we plot in Fig. 3 the compression curves of $\alpha, \omega$, and $\beta$ phases. For comparison, the experimental data from Ref. [26] are also shown in the figure. Clearly, our calculated $P-V$ equation of states are well consistent with the experimental measurement. The slight smallness of our calculated volumes compared to that of the experimental data can be attributed to the temperature effect. The experiment was performed at room temper- 


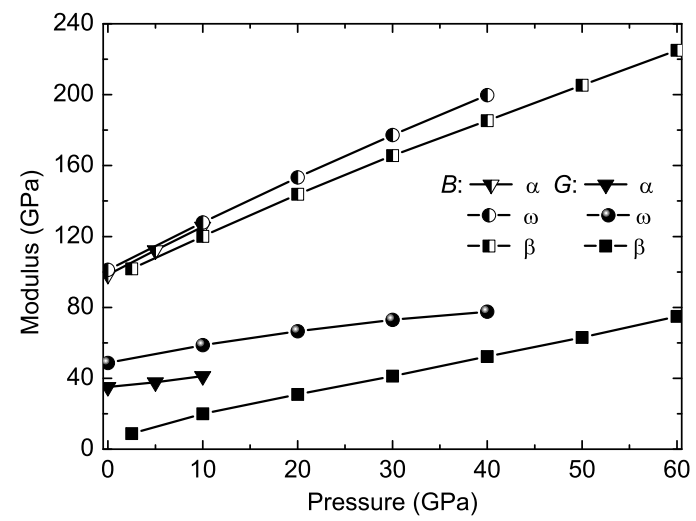

FIG. 5: Calculated moduli for TiZr alloy as a function of pressure. Solid lines are guides to the eyes.

ature, while our data are valid only at $0 \mathrm{~K}$. In Fig. 4, we compare the calculated lattice parameters of $\alpha$ and $\omega$ phases with experimental results [26]. Table 1 also lists the optimized structural parameters of TiZr alloy at some different pressures. In the whole pressure domain of 0-60 GPa, the predicted lattice parameters for both $\alpha$ and $\omega$ phases compare well with the experimental values, which supplies the safeguard for our following study of mechanical and elastic properties of TiZr alloy under pressure.

\section{Elasticity}

Elastic constants can measure the resistance and mechanical features of crystal to external stress or pressure, thus describing the stability of crystals against elastic deformation. In present work, elastic constants for TiZr alloy in $\alpha, \omega$, and $\beta$ phases at different pressures are calculated through applying stress tensors with various small strains onto the optimized structures. The strain amplitude $\delta$ is varied in steps of 0.006 from $\delta=-0.036$ to 0.036. Results are presented in Table 1. Obviously, the mechanical stability of $\alpha$ and $\omega$ TiZr at 0 GPa and at some typical finite pressures can be predicted from the elastic constants data. But the elastic constants of $\beta$ phase at $0 \mathrm{GPa}$ illustrate that the cubic phase is mechanically unstable. We notice that the $\beta$ phase metals Ti and $\mathrm{Zr}$ are also mechanically unstable at $0 \mathrm{GPa}$ [16, 43]. Along with the increase of pressure from 0 GPa to 60 GPa, the value of $C_{11}-C_{12}$ increases near linearly from $-5.3 \mathrm{GPa}$ to $146.3 \mathrm{GPa}$ for TiZr. Fitting the curve (not shown) of the pressure behavior of $C_{11}-C_{12}$ by polynomial function, we find that the value of $C_{11}-C_{12}$ becomes positive at $P \geq 2.19 \mathrm{GPa}$. In fact, at $P=2.5 \mathrm{GPa}$ our calculated $C_{11}, C_{12}$, and $C_{44}$ equal to $102.9,101.2$, and 25.6 GPa, respectively, which explicitly indicate the elastically stable of bcc TiZr under this pressure.

After obtaining elastic constants, the polycrystalline

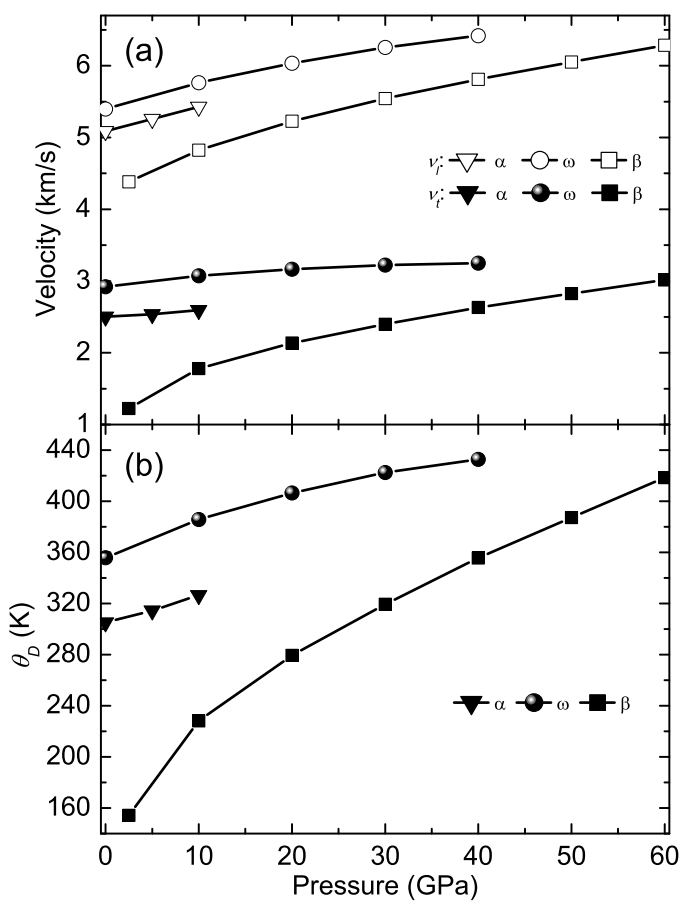

FIG. 6: Transverse and longitudinal sound velocities (a) and Debye temperature (b) for TiZr alloy as a function of pressure. Solid lines are guides to the eyes.

bulk modulus $B$ and shear modulus $G$ are calculated from the Voigt-Reuss-Hill (VRH) approximations [44]. Results of TiZr alloy in $\alpha, \omega$, and $\beta$ phases are shown in Fig. 5. The deduced bulk moduli from VRH approximations for all three phases at $0 \mathrm{GPa}$ turn out to be very close to that obtained from the EOS fitting, which indicates that our calculations are consistent and reliable. Along with the increasing of pressure, both bulk modulus $B$ and shear modulus $G$ increase near linearly for all three phases. The increasing rates of $B$ for all three phases are apparently larger than that of $G$. It is well known that the shear modulus $G$ represents the resistance to plastic deformation, while the bulk modulus $B$ can represent the resistance to fracture. A high (low) $B / G$ value is responsible for the ductility (brittleness) of polycrystalline materials. The critical value to separate ductile and brittle materials is about 1.75 [45]. Using the calculated values of bulk modulus $B$ and shear modulus $G$ for TiZr alloy, the $B / G$ values for $\alpha$ phase increase from 2.80 to 3.04 under pressure from $0 \mathrm{GPa}$ to $10 \mathrm{GPa}$, for $\omega$ phase increase from 2.08 to 2.57 upon compression from $0 \mathrm{GPa}$ to $40 \mathrm{GPa}$, and for $\beta$ phase decrease from 11.57 to 3.01 under pressure from $2.5 \mathrm{GPa}$ to $60 \mathrm{GPa}$. These results indicate that transit to $\omega$ phase will lead to less ductility and further transit to $\beta$ phase will result in more predominance of ductility. Although the large $B / G$ values for $\beta$ TiZr at pressure range of $0-30 \mathrm{GPa}$ can not be achieved in experiment at room temperature, the $B / G$ value of about 3.5 for $\beta$ phase at $40 \mathrm{GPa}$ indicates that 


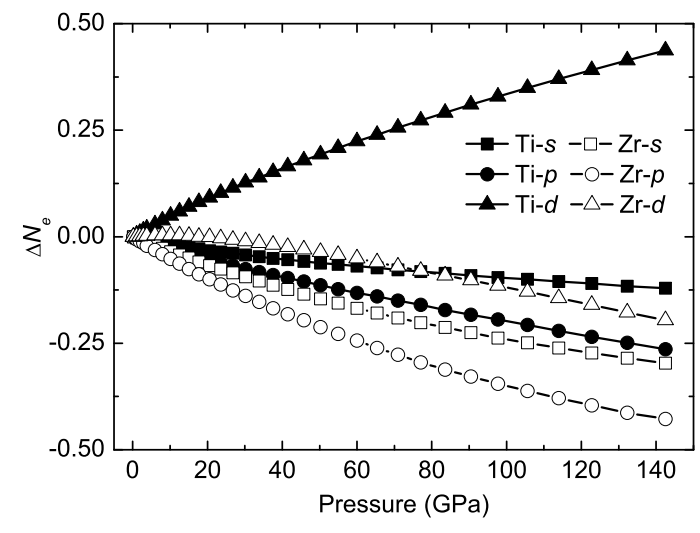

FIG. 7: Number of electrons on $s, p$, and $d$ orbitals for TiZr alloy as a function of pressure, relative to their values at 0 pressure. The numbers of $s$ electrons $\left(N_{s}\right), p$ electrons $\left(N_{p}\right)$, and $d$ electrons $\left(N_{d}\right)$ at $P=0 \mathrm{GPa}$ for Ti atom are 2.469, 6.417, and 3.012, respectively, for $\mathrm{Zr}$ atom are 2.565, 6.413, and 3.012 , respectively.

the $\beta$ phase TiZr alloy possess the biggest ductility. For $\alpha \mathrm{Zr}$, the $B / G$ value of 2.63 at ambient condition can be derived from the elastic data in Ref. [46]. Therefore, TiZr alloy is rather ductile and its ductility is more predominant than that of element Zr. We hope that our calculated elastic constants and elastic moduli can be illustrative in the realistic application of the mechanical data for TiZr alloy.

Elastic wave velocities provide important information about the behavior of materials before phase transition. The transverse and longitudinal elastic wave velocities of the polycrystalline materials can be calculated through relations of $v_{t}=\sqrt{G / \rho}$ ( $\rho$ is the density) and $v_{l}=\sqrt{(3 B+4 G) / 3 \rho}$, respectively. The average wave velocity in the polycrystalline materials is approximately given as $v_{m}=\left[(1 / 3)\left(2 / v_{t}^{3}+1 / v_{l}^{3}\right)\right]^{-1 / 3}$. Using the relation $\theta_{D}=\left(h / k_{B}\right)(3 n / 4 \pi \Omega)^{1 / 3} v_{m}$, the Debye temperature $\left(\theta_{D}\right)$ can be obtained. The calculated results of transverse and longitudinal elastic wave velocity are plotted in Fig. 6(a) and the Debye temperature in Fig. 6(b). Increasing behaviors of elastic wave velocities and $\theta_{D}$ under pressure are obvious. Transiting from $\alpha$ to $\omega$ at around $10 \mathrm{GPa}$ (experimental transition pressure), TiZr alloy exhibit abrupt increasing feature for both transverse and longitudinal elastic wave velocities: from 5.43 to 5.76 $\mathrm{km} / \mathrm{s}$ for $v_{t}$ and from 2.60 to $3.07 \mathrm{~km} / \mathrm{s}$ for $v_{l}$. In study of element Zr, Liu et al. [46] observed similar behaviors of elastic wave velocities by using ultrasonic interferometry in conjunction with synchrotron x-ray radiation. From Fig. 6(a), we find that the elastic wave velocities will decrease upon the $\omega$ to $\beta$ transition at high pressure. The Debye temperature also shows this kind of increase first (at $\alpha$ to $\omega$ transition) and decrease later (at $\omega$ to $\beta$ transition) behavior upon compression.

For discussion of pressure induced $s$ - $d$ electron transfer for TiZr alloy, we have performed Mulliken population analysis [47] of its $\beta$ phase and then plot in Fig. 7 the variation of the number of electrons on $s, p$, and $d$ orbitals with increasing pressure. Clearly, only the $N_{d}$ of $\mathrm{Ti}$ atom increases with pressure. Arithmetic average of $N_{d}$ for $\mathrm{Ti}$ and $\mathrm{Zr}$ atoms also possesses obvious pressuredependent increasing behavior. Increase of $d$-band occupancy will stabilize $\beta$ phase of TiZr alloy under pressure. However, we find that the $s p$ electrons for Ti atom and the spd electrons for $\mathrm{Zr}$ atom all decrease upon compression up to $143 \mathrm{GPa}$. No increasing behavior of $N_{s}$ has been observed. This fact is different from $\mathrm{Ti}$ 43] and $\mathrm{Zr}$ [48], where it was found that the $N_{s}$ in both of these two elemental metals start to increase when the pressure exceeds about 70 and $100 \mathrm{GPa}$, respectively. Thus, the $s$ - $d$ electron transfer behaviors for TiZr alloy are different from its archetype metals.

\section{Phonon dispersion}

To our knowledge, although plenty of attentions have been paid on the phonon dispersions, thermodynamics, and $P-T$ phase diagrams of $\mathrm{Ti}[42,49]$ and $\mathrm{Zr}[16-18$, 50], no experimental and theoretical phonon dispersion results have been published for equiatomic TiZr alloy. In this subsection and the following subsection, we want to present our calculated results of phonon dispersion curves, thermodynamics, and $P-T$ phase diagram for TiZr alloy.

We use the supercell approach [51] and the small displacement method as implemented in the FROPHO code 52 to calculate the phonon curves in the BZ and the corresponding phonon density of states (DOS). In the interpolation of the force constants for the phonon dispersion curve calculations, $5 \times 5 \times 5,3 \times 3 \times 1$, and $5 \times 5 \times 5$ $k$-point meshes are used for $\alpha 3 \times 3 \times 3, \omega 3 \times 3 \times 3$, and $\beta$ $4 \times 4 \times 4$ supercells, respectively. The forces induced by small displacements are calculated by VASP.

The calculated phonon dispersion curves and the phonon DOS of $\alpha, \omega$, and $\beta \mathrm{TiZr}$ alloy at $0 \mathrm{GPa}$ are displayed in Fig. 8. Overall speaking, behaviors of the phonons in the BZ for $\alpha$ and $\omega$ TiZr alloy are similar to that of metal $\mathrm{Ti}$ [42] and $\mathrm{Zr}$ [16]. Same with $\mathrm{Zr}$, the $\omega$ phonons for TiZr alloy are stiffer along the $c$ axis than in the basal plane due to the low $c / a$ ratio. The $\alpha$ phonons along [001] direction for TiZr alloy are softer than that of metal Ti and Zr. Compared with $\beta$ Ti and $\beta \mathrm{Zr}$, phonons in the $\mathrm{BZ}$ for $\beta$ phase of TiZr alloy exhibit different behaviors. The unstable phonon branch along [110] direction in the two metals transfer to show stable and stiff feature in the alloy. On the contrary, the phonons along [001] direction are stable for metals but unstable for the alloy. These informations are important in analyzing phase transformation. The stable nature of phonon branch along [110] direction for $\beta$ TiZr alloy may responsible for the difficulties of $\alpha \rightarrow \beta$ transition under high temperature and the $\omega \rightarrow \beta$ transition under high pressure as indicated in experiments $24,26,27]$. 

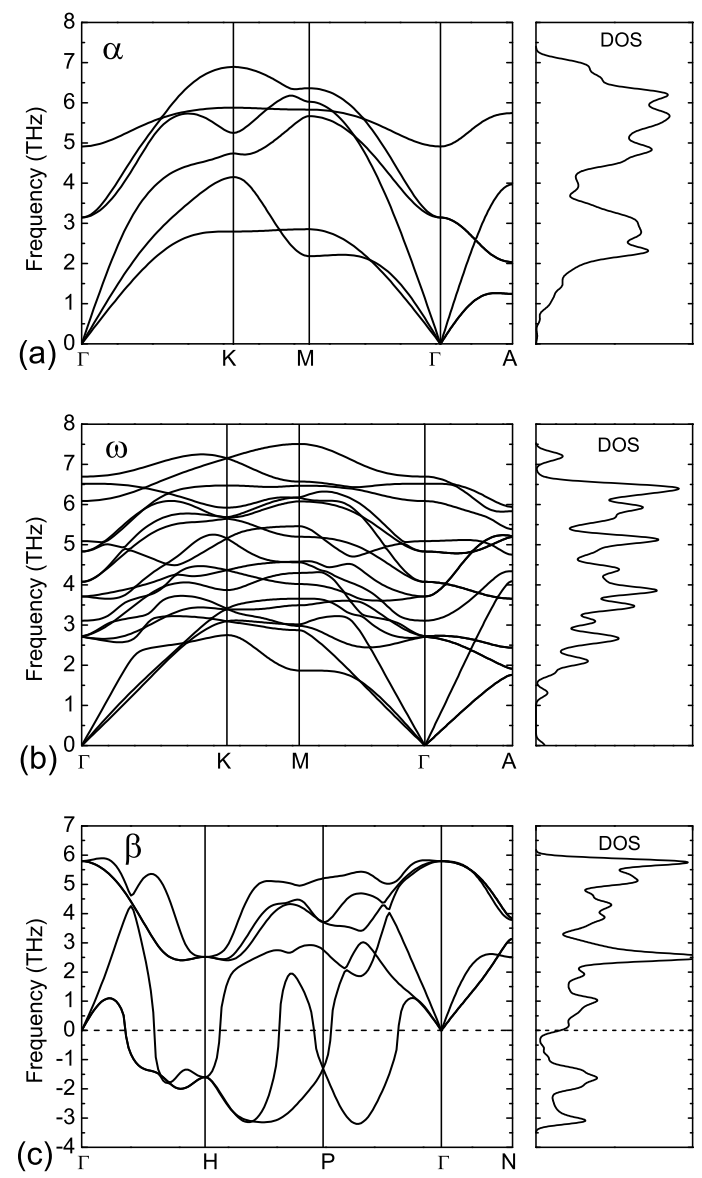

FIG. 8: Phonon dispersion curves and phonon DOS of $\alpha, \omega$, and $\beta$ TiZr alloy.

\section{E. Thermodynamic properties and $P-T$ phase diagram}

Thermodynamic properties of equiatomic TiZr alloy can be determined by phonon calculation using the quasiharmonic approximation (QHA) [53, 54] or by the quasiharmonic Debye model [55]. Within these two models, the Gibbs free energy $G(T, P)$ is written as

$$
G(T, P)=F(T, V)+P V .
$$

Here, $F(T, V)$ is the Helmholtz free energy at temperature $T$ and volume $V$ and can be expressed as

$$
F(T, V)=E(V)+F_{v i b}(T, V)+F_{e l}(T, V),
$$

where $E(V)$ is the ground-state total energy, $F_{v i b}(T, V)$ is the vibrational energy of the lattice ions and $F_{e l}(T, V)$ is the thermal electronic contribution.

Under QHA, the $F_{v i b}(T, V)$ can be calculated by

$$
F_{v i b}(T, V)=k_{B} T \int_{0}^{\infty} g(\omega) \ln \left[2 \sinh \left(\frac{\hbar \omega}{2 k_{B} T}\right)\right] d \omega
$$

where $\omega$ represents the phonon frequencies and $g(\omega)$ is the phonon DOS. This formula requests positive results of phonon DOS. So it is not suitable for dynamically unstable phases. Instead, the vibration energy for phases with imaginary phonon frequencies can be estimated by the Debye model

$$
F_{v i b}(T, V)=\frac{9}{8} k_{B} \theta_{D}+k_{B} T\left[3 \ln \left(1-e^{-\frac{\theta_{D}}{T}}\right)-D\left(\frac{\theta_{D}}{T}\right)\right]
$$

where $\frac{9}{8} k_{B} \theta_{D}$ is zero-point energy due to lattice ion vibration at $0 \mathrm{~K}$ and $D\left(\theta_{D} / T\right)$ the Debye integral written as $D\left(\theta_{D} / T\right)=3 /\left(\theta_{D} / T\right)^{3} \int_{0}^{\theta_{D} / T} x^{3} /\left(e^{x}-1\right) d x$. Detailed computation scheme of Debye model please see Ref. [55].

$F_{e l}$ in Eq. (2) can be obtained from the energy and entropy contributions, i.e., $E_{\text {ele }}-T S_{\text {ele }}$. The electronic entropy $S_{e l e}$ is of the form

$$
S_{\text {ele }}(V, T)=-k_{B} \int n(\varepsilon, V)[f \ln f+(1-f) \ln (1-f)] d \varepsilon,
$$

where $n(\varepsilon)$ is electronic DOS and $f$ is the Fermi-Dirac distribution. The energy $E_{\text {ele }}$ due to the electron excitations takes the following form:

$$
E_{\text {ele }}(V, T)=\int n(\varepsilon, V) f \varepsilon d \varepsilon-\int^{\varepsilon_{F}} n(\varepsilon, V) \varepsilon d \varepsilon,
$$

where $\varepsilon_{F}$ is the Fermi energy.

Using QHA and Debye model, we calculated the Gibbs free energy $(G)$, entropy $(S)$, and specific heat at constant volume $\left(C_{V}\right)$ for $\alpha, \omega$, and $\beta$ phases of TiZr alloy at their equilibrium volumes. As shown in Fig. 9, the Gibbs free energy and entropy calculated by QHA are almost identical to those obtained by employing the Debye model for $\alpha$ phase, while the differences between these two schemes for $\omega$ phase is slightly large, especially for high-temperature domain. Although the phonon calculation is more reliable in general analysis, some nonharmonic terms, which is important for $\omega$ phase, possibly having been ignored in the QHA method. From Fig. $9(\mathrm{a})$, cross between $\alpha$ and $\omega$ curves by Debye calculations readily gives phase transition temperature of $680 \mathrm{~K}$. A phase transition temperature of $940 \mathrm{~K}$ for $\alpha \rightarrow \beta$ also can be seen. So, we can use Debye model to obtain the $P-T$ phase diagram by changing the pressure $P$ (see Fig. 10). However, there is no cross between $\alpha$ and $\omega$ curves using phonon calculations. This need more works to clarify. Figure 9(b) shows the entropy results. While the entropy of $\omega$ phase is lower than $\alpha$ phase, the results of $\beta$ phase are higher than $\alpha$ phase in all considered temperature domain. The specific heat at constant volume $\left(C_{V}\right)$ can be directly calculated by $T\left(\frac{\partial S}{\partial T}\right)_{V}$. Results are presented in Fig. 9(c), using which one can estimate the Debye temperature. Actually, our phonon calculations indicate that the zero-point energies for $\alpha$ and $\omega$ phases at $P=0$ $\mathrm{GPa}$ are 27.02 and $28.00 \mathrm{meV}$, respectively. So, the Debye temperature values from phonon calculations equal to 278.6 and $288.9 \mathrm{~K}$, respectively. The Debye model 

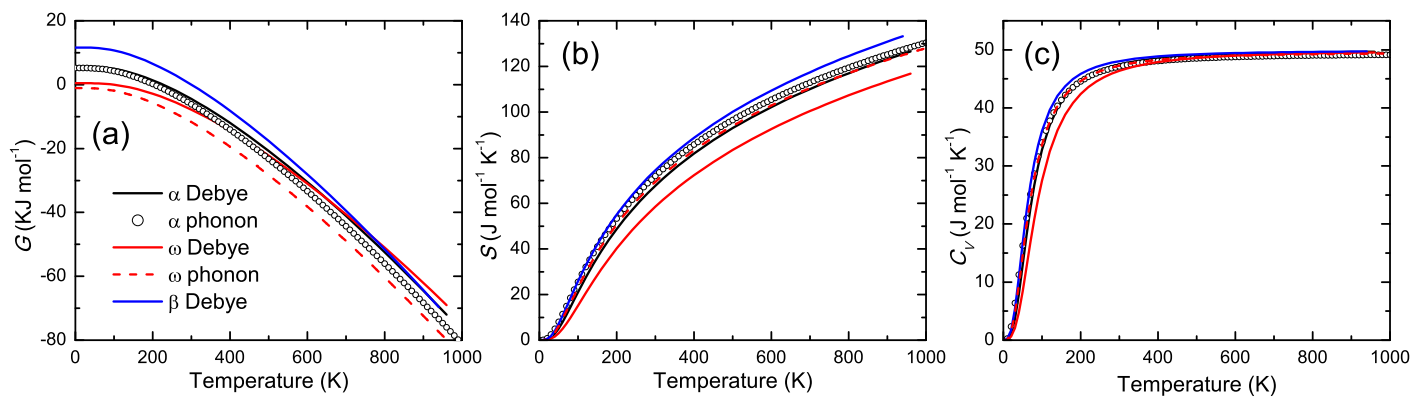

FIG. 9: Temperature dependences of (a) Gibbs free energy, (b) entropy, and (c) specific heat at constant volume $\left(C_{V}\right)$ for $\alpha$, $\omega$, and $\beta$ phases of TiZr alloy.

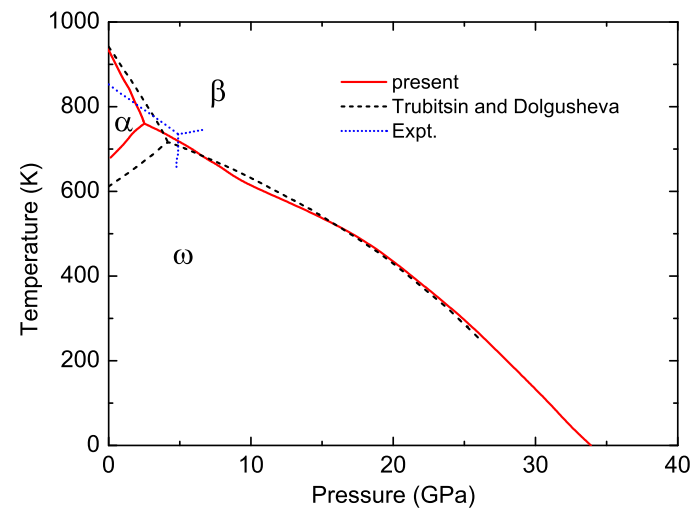

FIG. 10: Calculated $P-T$ phase diagram of TiZr alloy. For comparison, we also plot in figure the experimental data [24] and the theoretical results from Ref. [29].

gives $\theta_{D}=303.6$ and $369.0 \mathrm{~K}$ for $\alpha$ and $\omega$ phases, respectively. So, the Debye model gives closer values than the phonon calculations with respect to the Debye temperature values of 305.1 and $355.6 \mathrm{~K}$ computed by elastic constants (see Fig. 7).

We present in Fig. 10 the $P-T$ phase diagram of equiatomic TiZr alloy calculated by the Debye model. A triple point $\left(P_{\mathrm{tr}}=2.5 \mathrm{GPa}, T_{\mathrm{tr}}=760 \mathrm{~K}\right)$, comparable to experimental results $\left(P_{\mathrm{tr}}=4.9 \pm 0.3 \mathrm{GPa}, T_{\mathrm{tr}}=733 \pm 30\right.$ K) 24] and previous theoretical values $\left(P_{\mathrm{tr}}=4.2 \mathrm{GPa}\right.$, $\left.T_{\mathrm{tr}}=720 \mathrm{~K}\right)[29]$, is predicted. The $\omega \rightarrow \alpha$ and $\alpha \rightarrow \beta$ transition temperatures of 680 and $940 \mathrm{~K}$ are close to previous Debye-Grüneisen values (610 and $943 \mathrm{~K}$, respectively) [29]. Detailed understanding of the $P-T$ phase diagram please see Ref. [29].

\section{CONCLUSION}

In summary, structure, phase transition, elasticity, phonon, and thermodynamics of the equiatomic TiZr al- loy have been studied by means of the first-principles DFT-PBE method. The calculated lattice parameters and equations of state for $\alpha$ and $\omega$ phases are in good agreement with experiments. We have found that the $\omega$ phase is most stable at $0 \mathrm{GPa}$ and the $\omega \rightarrow \beta$ transition was calculated to occur at around $33.9 \mathrm{GPa}$. The elastic constants of $\alpha$ phase TiZr alloy at $P=0 \mathrm{GPa}$ coincide well with previous calculations. Mechanical stability of $\alpha$ and $\omega$ phases were predicted and the $\beta$ phase is mechanically unstable at zero pressure. Our elastic constants indicate that the $\beta$ phase will become mechanically stable upon compression. Under pressure, both bulk modulus $B$ and shear modulus $G$ increase near linearly for all three phases. Increasing behaviors of elastic wave velocities and Debye temperature under pressure have also been predicted. It was found that elastic wave velocities and Debye temperature increase first (at $\alpha$ to $\omega$ transition) and decrease later (at $\omega$ to $\beta$ transition) abruptly upon compression. The Mulliken population analysis showed that either $s p$ electrons of $\mathrm{Ti}$ atom or spd electrons of $\mathrm{Zr}$ atom transfer to the Ti- $d$ orbital with increasing pressure.

In phonon dispersion study, the dynamic stable nature for $\alpha$ and $\omega$ phases are observed. The unstable modes of $\beta$ phase TiZr alloy along [001] and [111] directions have been shown and these imaginary vibration branches are different from elemental $\mathrm{Ti}$ and $\mathrm{Zr}$. Based on phonon calculations and also the Debye model, thermodynamic properties of Gibbs free energy, entropy, and specific heat at constant volume as well as the $P-T$ phase diagram for TiZr alloy have been presented.

\section{Acknowledgments}

We thank Yu Yang for useful discussion. This work was supported by NSFC under Grant Nos. 11104170, 51071032, and 11074155, the Foundations for Development of Science and Technology of China Academy of Engineering Physics under Grant No. 2009B0301037. 
[1] H. Ikehata, N. Nagasako, T. Furuta, A. Fukumoto, K. Miwa, T. Saito, Phys. Rev. B 70 (2004) 174113.

[2] W. Liu, B. Li, L. Wang, J. Zhang, Y. Zhao, Phys. Rev. B 76 (2007) 144107.

[3] M.T. Pérez-Prado, A.P. Zhilyaev, Phys. Rev. Lett. 102 (2009) 175504.

[4] Y.K. Vohra, P.T. Spencer, Phys. Rev. Lett. 86 (2001) 3068.

[5] T.R.G. Kutty, K. Ravi, C. Ganguly, J. Nucl. Mater. 265 (1999) 91.

[6] J.C. Duthie, D.G. Pettifor, Phys. Rev. Lett. 38 (1977) 564.

[7] H.L. Skriver, Phys. Rev. B 31 (1985) 1909.

[8] V.A. Zilbershtein, N.P. Chistotina, A.A. Zharov, N.S. Grishina, E.I. Estrin, Fiz. Met. Metalloved. 39 (1975) 445.

[9] S.K. Sikka, Y.K. Vohra, R. Chidambaram, Prog. Mater. Sci. 27 (1982) 245.

[10] H. Xia, S.J. Duclos, A.L. Ruoff, Y.K. Vohra, Phys. Rev. Lett. 64 (1990) 204.

[11] Y. Zhao, J. Zhang, C. Pantea, J. Qian, L.L. Daemen, P.A. Rigg, R.S. Hixson, G.T. Gray, Y. Yang, L. Wang, Y. Wang, T. Uchida, Phys. Rev. B 71 (2005) 184119.

[12] H. Xia, A.L. Ruoff, Y.K. Vohra, Phys. Rev. B 44 (1991) 10374.

[13] Y. Akahama, M. Kobayashi, H. Kawamura, J. Phys. Soc. Jpn. 59 (1990) 3843.

[14] Y. Akahama, M. Kobayashi, H. Kawamura, J. Phys. Soc. Jpn. 60 (1991) 3211.

[15] Y.S. Zhao, J.Z. Zhang, Appl. Phys. Lett. 91 (2007) 201907.

[16] B.T. Wang, P. Zhang, H.Y. Liu, W.D. Li, P. Zhang, J. Appl. Phys. 109 (2011) 063514.

[17] Y.J. Hao, L. Zhang, X.R. Chen, L.C. Cai, Q. Wu, D. Alfè, Phys. Rev. B 78 (2008) 134101.

[18] I. Schnell, R.C. Albers, J. Phys.: Condens. Matter 18 (2006) 1483.

[19] R. Ahuja, J.M. Wills, B. Johansson, O. Eriksson, Phys. Rev. B 48 (1993) 16269.

[20] Y. Akahama, H. Kawamura, T. LeBihan, Phys. Rev. Lett. 87 (2001) 275503.

[21] D. Errandonea, Y. Meng, M. Somayazulu, D. Häusermann, Physica B 355 (2005) 116.

[22] Z.G. Mei, S.L. Shang, Y. Wang, Z.K. Liu, Phys. Rev. B 79 (2009) 134102.

[23] A.K. Verma, P. Modak, R.S. Rao, B.K. Godwal, R. Jeanloz, Phys. Rev. B 75 (2007) 014109.

[24] I.O. Bashkin, A.Yu. Pagnuev, A.F. Gurov, V.K. Fedotov, G.E. Abrosimova, E.G. Ponyatovskii, Phys. Solid State 42 (2000) 170.

[25] I.O. Bashkin, V.G. Tissen, M.V. Nefedova, A. Schiwek, W.B. Holzapfel, E.G. Ponyatovsky, JETP Lett. 73 (2001) 80.

[26] I.O. Bashkin, V.K. Fedotov, M.V. Nefedova, V.G. Tissen, E.G. Ponyatovsky, A. Schiwek, W.B. Holzapfel, Phys.
Rev. B 68 (2003) 054401.

[27] V.V. Aksenenkov, V.D. Blank, B.A. Kulnitskiy, E.I. Estrin, Phys. Met. Metalloved. 69 (1990) 154.

[28] V.P. Dmitriev, L. Dubrovinsky, T. Le Bihan, A. Kuznetsov, H.P. Weber, E.G. Poniatovksy, Phys. Rev. B 73 (2006) 094114.

[29] V.Yu. Trubitsin, E.B. Dolgusheva, J. Phys.: Condens. Matter 22 (2010) 465401; Phys. Solid State 53 (2) (2011) 223.

[30] P.E. Blöchl, Phys. Rev. B 50 (1994) 17953.

[31] G. Kresse, J. Furthmüller, Phys. Rev. B 54 (1996) 11169.

[32] J.P. Perdew, K. Burke, M. Ernzerhof, Phys. Rev. Lett. 77 (1996) 3865.

[33] H.J. Monkhorst, J.D. Pack, Phys. Rev. B 13 (1972) 5188.

[34] F. Birch, Phys. Rev. 71 (1947) 809.

[35] G. Jomard, L. Magaud, A. Pasturel, Phil. Mag. 77 (1998) 67.

[36] A. Landa, P. Söderlind, P. E. A. Turchi, J. Alloys Compd. 478 (2009) 103.

[37] S.A. Ostanin, V. Yu. Trubitsin, Phys. Stat. Sol. (b) 201 (1997) R9; Phys. Rev. B 57 (1998) 13485.

[38] F. Jona, P. M. Marcus, J. Phys.: Condens. Matter 15 (2003) 5009.

[39] F. Jona, P.M. Markus, Phys. Stat. Sol. (b) 242 (2005) 3077.

[40] G.B. Grad, P. Blaha, J. Luitz, K. Schwarz, A. Fernández Guillermet, S.J. Sferco, Phys. Rev. B 62 (2000) 12743.

[41] C.W. Greef, Modelling Simul. Mater. Sci. Eng. 13 (2005) 1015.

[42] Z.G. Mei, S.L. Shang, Y. Wang, Z.K. Liu, Phys. Rev. B 80 (2009) 104116.

[43] Q.M. Hu, S. Lu, R. Yang, Phys. Rev. B 78 (2008) 052102.

[44] R. Hill, Phys. Phys. Soc. London 65, 349 (1952).

[45] S. F. Pugh, Phil. Mag. 45 (1954) 823.

[46] W. Liu, B. Li, L. Wang, J. Zhang, Y. Zhao, J. Appl. Phys. 104 (2008) 076102.

[47] R.S. Mulliken, J. Chem. Phys. 23 (1955) 1833.

[48] S. Zhang, Y. Zhu, X. Zhang, S. Zhang, L. Qi, R. Liu, Comp. Mater. Sci. 50 (2010) 179.

[49] P. Souvatzis, O. Eriksson, M. I. Katsnelson, and S. P. Rudin, Phys. Rev. Lett. 100, 095901 (2008).

[50] A. Heiming, W. Petry, J. Trampenau, M. Alba, C. Herzig, H. R. Schober, and G. Vogl, Phys. Rev. B 43, 10948 (1991).

[51] K. Parlinski, Z-.Q. Li, and Y. Kawazoe, Phys. Rev. Lett. 78, 4063 (1997).

[52] A. Togo, F. Oba, and I. Tanaka, Phys. Rev. B 78 (2008) 134106.

[53] A. Siegel, K. Parlinski, and U. D. Wdowik, Phys. Rev. B 74, 104116 (2006).

[54] P. Zhang, B.-T. Wang, and X.-G. Zhao, Phys. Rev. B 82, 144110 (2010).

[55] A. A. Blanco, E. Francisco, and V. Luana, Comput. Phys. Commun. 158 (2004) 57. 